\title{
Evaluación del desempeño de los docentes de la Facultad de Ingeniería de Minas de la Universidad Nacional del Centro del Perú
}

\section{Performance evaluation of teachers of the Faculty of Engineering of Mines of the Universidad Nacional del Centro del Perú}

\author{
Eusebio Zenon Castro Leon ${ }^{*}$ \\ ' Facultad de Ing. de Minas, Universidad Nacional del Centro del Perú, Huancayo, Perú
}

\section{RESUMEN}

El objetivo general fue describir el desempeño de los docentes de La facultad de Ingeniería de Minas de la Universidad Nacional del Centro del Perú. Este trabajo corresponde a un nivel descriptivo, no experimental. La población fue de: estudiantes (466), docentes (26), egresados (30), administrativos (4), autoridades (4). La técnica utilizada fue la encuesta, como instrumento de recolección de datos se utilizó el cuestionario de desempeño docente, que fue sometido a la prueba de confiabilidad de Alfa de Crombach. Algunos resultados del desempeño docente fueron: respecto al dominio de la asignatura $40,6 \%$ califica como muy bueno, en vocación 17,3 $\%$ como bueno, asistencia y puntualidad 14,2 \% como excelente, preocupación y comprensión de los problemas de los estudiantes 19,5\% como bueno. El análisis global se realizó considerando las siguientes dimensiones, facilidad del aprendizaje con 7 ítems, emocionalidad con 4 ítems; responsabilidad laboral con 3 ítems, relaciones interpersonales con sus estudiantes con 3 ítems y resultados de su labor educativa con 1 ítem. En términos generales, podemos considerar que de los 18 ítems evaluados, el $100 \%$ se encuentra con un calificación entre buena, muy buena y excelente. En conclusión, el desempeño docente de la Facultad de Ingeniería de Minas se encuentra con la calificación de buena, en todos los rubros evaluados.

Palabras clave: Emocionalidad, responsabilidad laboral, relaciones interpersonales, labor educativa.

\begin{abstract}
The general objective was to describe the performance of the teachers of the Faculty of Mines Engineering of the Universidad Nacional del Centro del Perú. This work corresponds to a descriptive level, not experimental. The population was: students (466), teachers (26), graduates (30), administrative (4), authorities (4). The technique used was the sampling, as a data collection instrument the teacher performane questionnaire was used, which was submitted to the reliability test of Crombachs Alpha. Some results of the teaching performance, regarding the mastery of the subject $40,6 \%$ qualifies as very good, in vocation $17,3 \%$ as good, attendance and punctuality $14,2 \%$ as excellent, concern and understanding of the problems of the students 19, $5 \%$ as good. The global analysis was carried out considering the following dimensions, ease of learning with 7 items, emotionality with 4 items; Labor responsibility with 3 items; interpersonal relationships with their students with 3 items and results of their educational work with 1 item. In general terms, we can consider that the 18 items under evaluation, $100 \%$ is rated as good, very good and excellent. In conclusion, the teaching performance of the Faculty of Mines Engineering is in the grade of good, in all the areas evaluated.
\end{abstract}

Keywords: Emotionality, labor responsability, interpersonal relationships, educational work.

Historial del artículo:

Recibido, 14 de abril de 2015; aceptado, 21 de octubre de 2016; disponible en línea, 25 de junio de 2017

* Docente de la Universidad Nacional del Centro del Perú.

Correo: zenoncastroleon3@hotmail.com 


\section{INTRODUCCIÓN}

Las Instituciones de Educación Superior y en especial las Universidades desempeñan un rol de suma importancia en la formación de recursos humanos del más alto nivel, en la creación, desarrollo, transferencia y adaptación de tecnología; y lo que hacen para responder adecuadamente a los requerimientos de la sociedad moderna se constituye en un imperativo estratégico para el desarrollo nacional. Las universidades son reconocidas cada vez más como un instrumento de desarrollo de ciudades, regiones y países, y están consideradas como un factor clave para incrementar la competitividad y calidad de vida. El desafío para las instituciones de Educación Superior es el de enfrentar un mundo en el cual los sistemas productivos están en permanente transformación (PNUD, 1995).

La evaluación de la calidad de las instituciones educativas, son cada vez más relevantes como una de las formas para elevar la calidad de éstas.

En ese contexto la excelencia docente es responsabilidad de toda la organización en su conjunto. El profesor tiene un importante grado de responsabilidad en lo que se refiere a su disposición, sus acciones y sus metas profesionales. No obstante, los directivos deben cumplir un relevante rol de auténtico líder facilitador, proporcionando las condiciones y oportunidades para la manifestación y desarrollo de la excelencia en el desempeño docente. Para lograr la excelencia en la universidad, es necesario evaluar el desempeño directivo y docente, teniendo presente que el centro del proceso evaluativo debería ser el logro de la excelencia, por lo tanto, el énfasis se encontraría en la auto evaluación durante el proceso para identificar donde están ubicadas las fortalezas y debilidades, con el propósito de diseñar o reformular estrategias que permitan un mejoramiento continuo del servicio educativo (Vidal, 2005).

El desempeño docente es lo puesto en práctica por el docente quien aporta sus conocimientos, capacidades, habilidades y actitudes, demostrando dominio de su temática, estrategias metodológicas, evaluación etc. Su misión es contribuir en su desarrollo humano sostenible. Contribuir, desde los espacios estructurados para la enseñanza sistemática, al desarrollo integral de las personas, incorporando sus dimensiones biológicas, afectivas, cognitivas, sociales y morales. Su función es mediar y asistir en el proceso por el cual el estudiante desarrolla sus conocimientos, capacidades, destrezas, actitudes y valores, en el marco de un comportamiento que valora a otros y respeta los derechos individuales y sociales (Cerdeña, 2007).

En tal perspectiva Valdez (2000) afirma que la evaluación del desempeño docente es "una actividad de análisis, compromiso y formación del profesorado, que valora y enjuicia la concepción, práctica, proyección y desarrollo de la actividad y de la profesionalización docente". La evaluación, quiérase o no, orienta la actividad educativa y determina el comportamiento de los sujetos, no sólo por los resultados que pueda ofrecer sino porque ella preestablece qué es lo deseable, qué es lo valioso, qué es lo que debe ser.

En este contexto el problema planteado fue, ¿̇Cómo es el desempeño de los docentes de la facultad de Ingeniería de Minas de la Universidad Nacional del Centro del Perú en Huancayo?. El objetivo general fue describir el desempeño de los docentes de La facultad de Ingeniería de Minas de la Universidad Nacional del Centro del Perú.

\section{MATERIAL Y MÉTODOS}

La sede del presente trabajo de Investigación fue la cuidad de Huancayo, Universidad Nacional del Centro del Perú, facultad de Ingeniería de Minas.

El tipo de investigación que corresponde el presente trabajo es la investigación Aplicada (Sánchez y Reyes, 1996) debido a que sus resultados nos permitirán conocer, evaluar y modificar lo actuado.

Este trabajo de investigación corresponde a una investigación de nivel descriptivo, no experimental, es decir aquella que comprende el registro, análisis e interpretación de la realidad problemática, composición o proceso de los fenómenos, el enfoque se realizó sobre condiciones o fenómenos dominantes en el presente, muchas veces lleva consigo algún tipo de comparación y con frecuencia responde a las siguientes preguntas: qué, quién y dónde.

El diseño de la investigación descriptiva transversal es la siguiente:

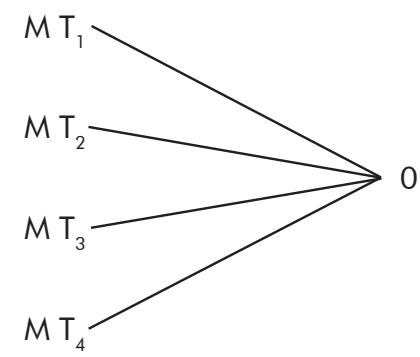

$M T_{1-4}$ : Representan las muestras de 4 etapas 0 momentos diferentes en el desarrollo de un fenómeno.

O : Representa la observación o medición que se realiza en estas edades. 
El método de la presente investigación corresponde al método deductivo, es decir que de aspectos generales se desglosan hasta llegar a los específicos

La población fue definida de la siguiente manera:

- Población estudiantil

- Población docentes

- Población de egresados

$: 30$

- Población de administrativos

$: 04$

- Población autoridades

$: 04$

La muestra se calculó en base a la siguiente fórmula:

$$
n=\frac{\left(Z_{1-\alpha / 2}+Z_{\beta}\right)^{2} \sigma^{2}}{\varepsilon^{2}}
$$

Donde:

$Z \alpha=$ Nivel de confianza empleado, que normalmente lo da el investigador, y generalmente se trabaja con niveles de confianza de 0,05 y 0,01

$Z \beta=$ Potencia de una prueba (error tipo II), valor que normalmente lo da el investigador.

$\sigma^{2}=$ Varianza, este valor debe ser calculado ya sea a través de un muestreo piloto o de estudios anteriores.

$\varepsilon^{2}=$ Error muestral lo establece generalmente el Investigador.

La muestra se calculó directamente con el Software estadístico MINITAB 14, determinando los siguientes tamaños de muestra:

- Estudiantes

$: 155$

- Docentes

$: 18$

- Egresados

: 30

- Administrativos

: 04

- Autoridades

: 04

Total

En el presente trabajo se utilizó la técnica de las encuestas, la que nos permitió obtener la información deseada, y dentro de ellas nuestros indicadores.

Como instrumento de recolección de datos se utilizó el cuestionario, que viene hacer la parte complementaria de la encuesta, posteriormente este cuestionario fue sometido a la prueba de confiabilidad de Alfa de Crombach, a través del uso del paquete estadístico caso específico del SPSS-14. Así mismo el instrumento utilizado fue el instrumento para desempeño docente.

\section{RESULTADOS}

\section{Evaluación del desempeño docente:}

Facilidad del aprendizaje:

La figura 1 muestra los resultados obtenidos del desempeño docente, rubro dominio de la asignatura, se nota que entre las categorías de muy bueno, bueno y excelente, abarcan casi la totalidad de las frecuencias, lo que nos indica, que los docentes de dicha facultad dominan sus cátedras.

La figura 2 nos muestra que entre el rubro bueno y muy bueno pasan del $60 \%$ del total de encuestados, esto significa que el desempeño docente en lo que se refiere a efectuar su clase entretenida, amena e interesante, la mayoría de profesores de La facultad

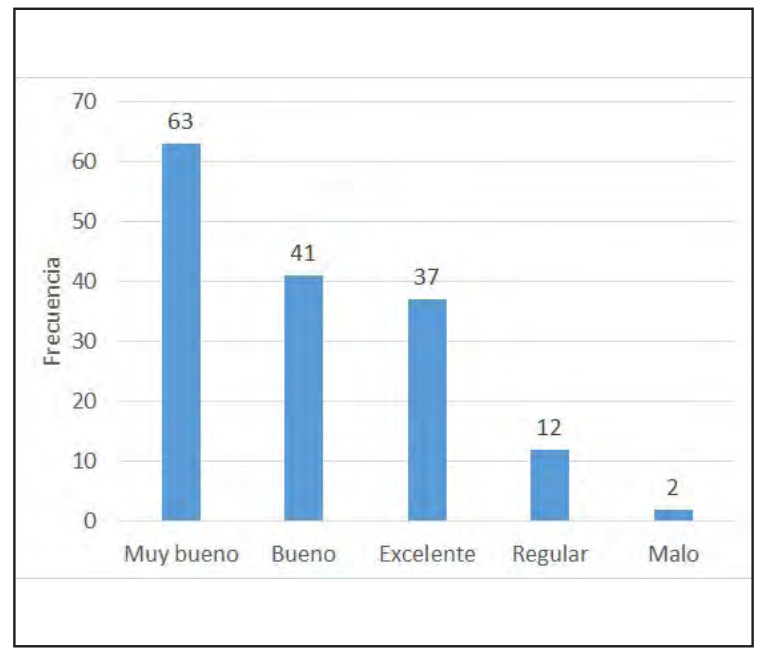

Figura 1.Dominio de la asignatura que imparte el docente.

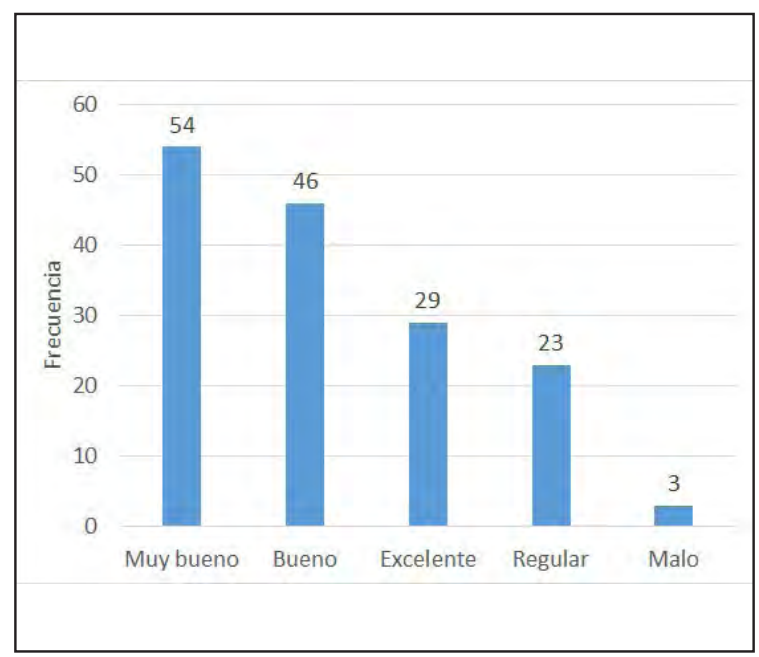

Figura 2. Capacidad de hacer su materia entretenida e interesante. 


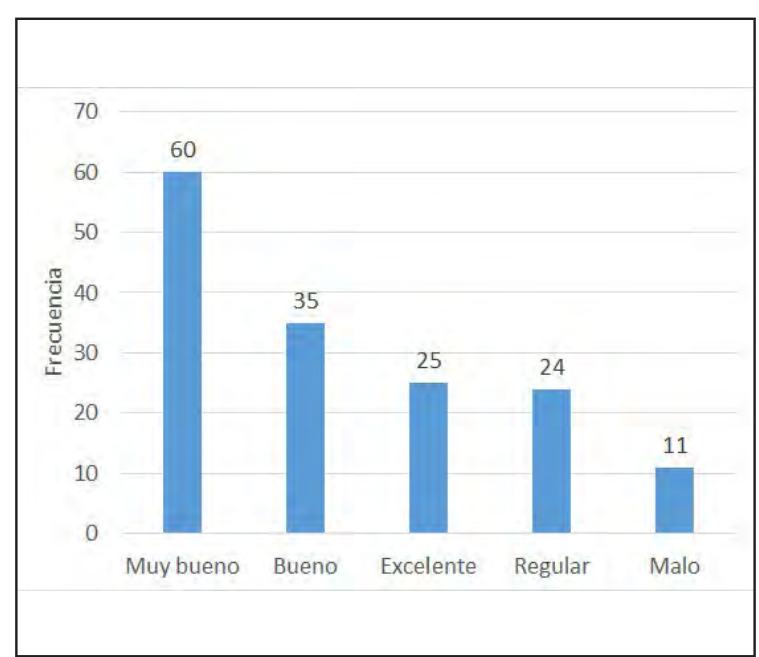

Figura 3. Comunicación con los estudiantes de la facultad de Ingeniería de Minas.

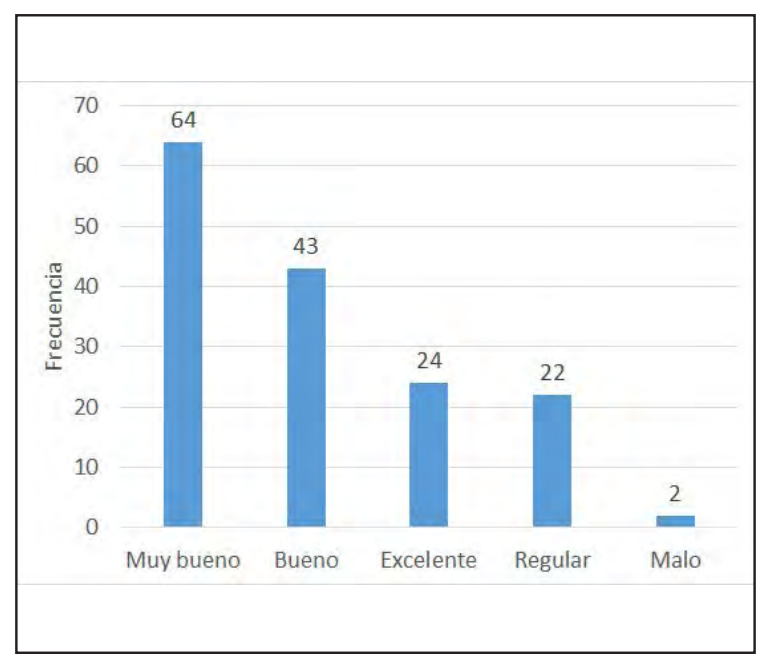

Figura 4.Capacidad de planificar adecuadamente el proceso docente - educativo.

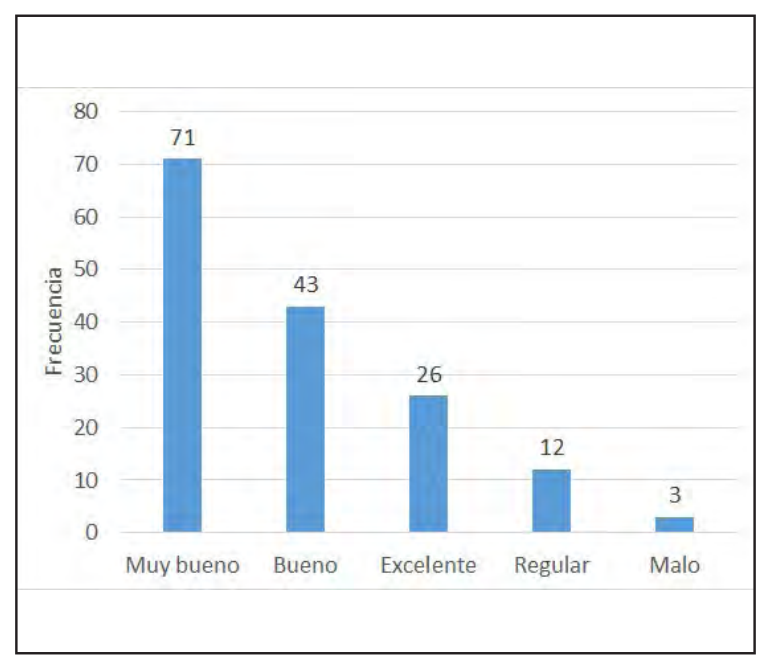

Figura 5. Utilización de recursos y materiales.

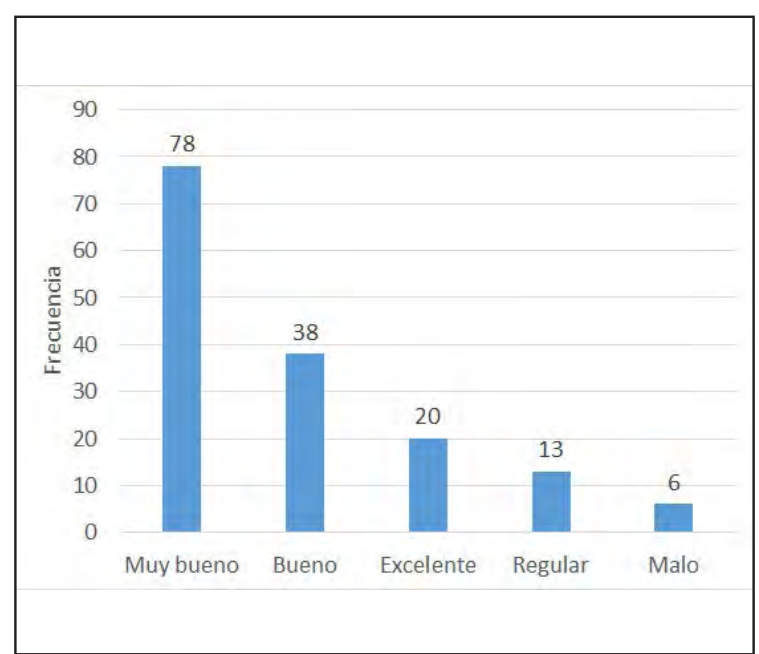

Figura 6.Grado de información sobre la marcha del aprendizaje de sus estudiantes.

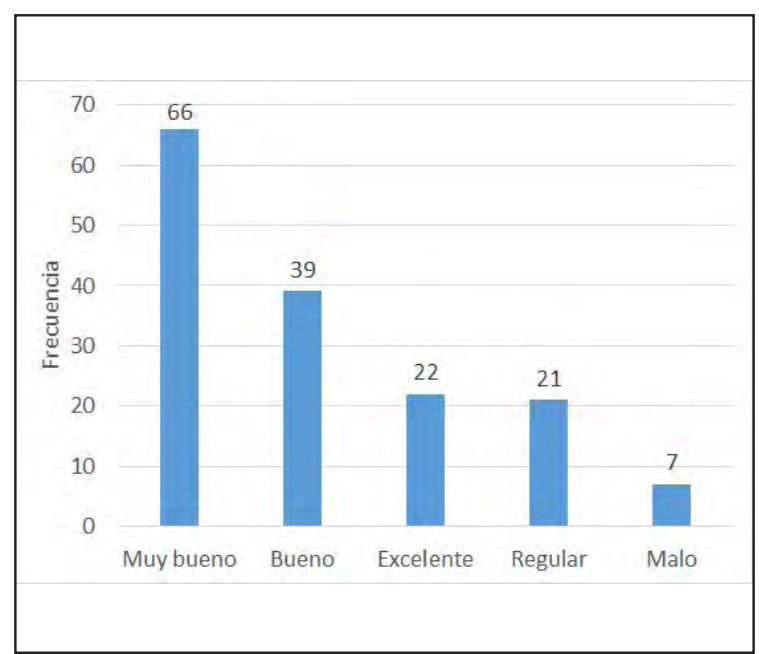

Figura 7.Capacidad para crear un ambiente favorable para que el estudiante conozca sus derechos y responsabilidades.

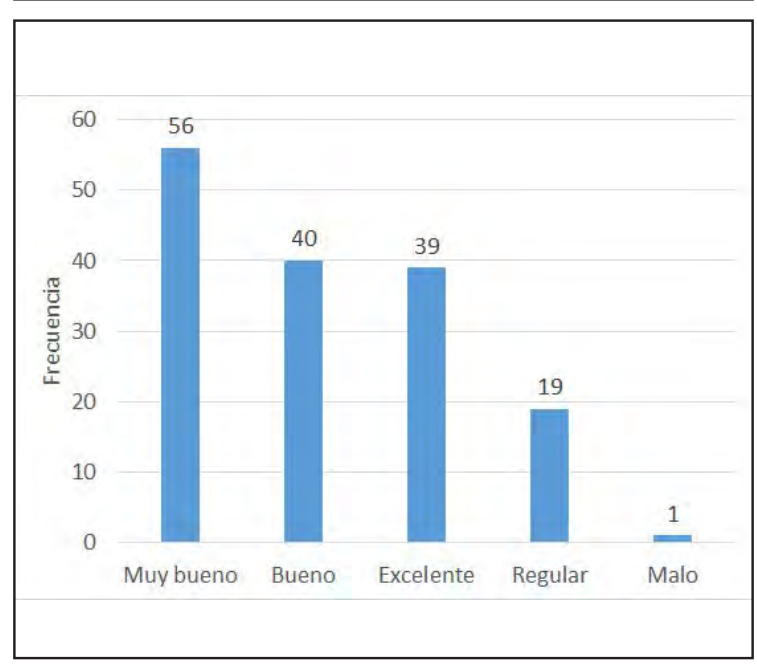

Figura 8. Vocación. 


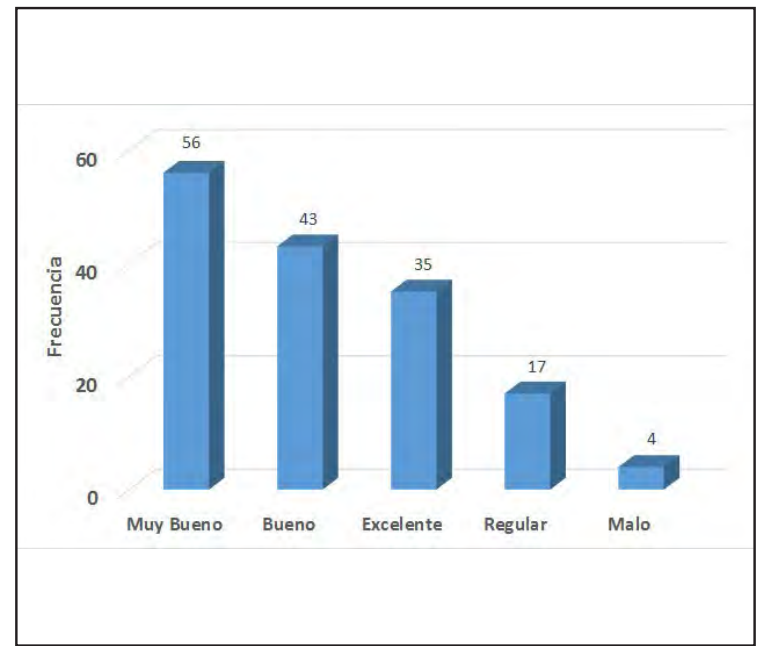

Figura 9. Autoestima.

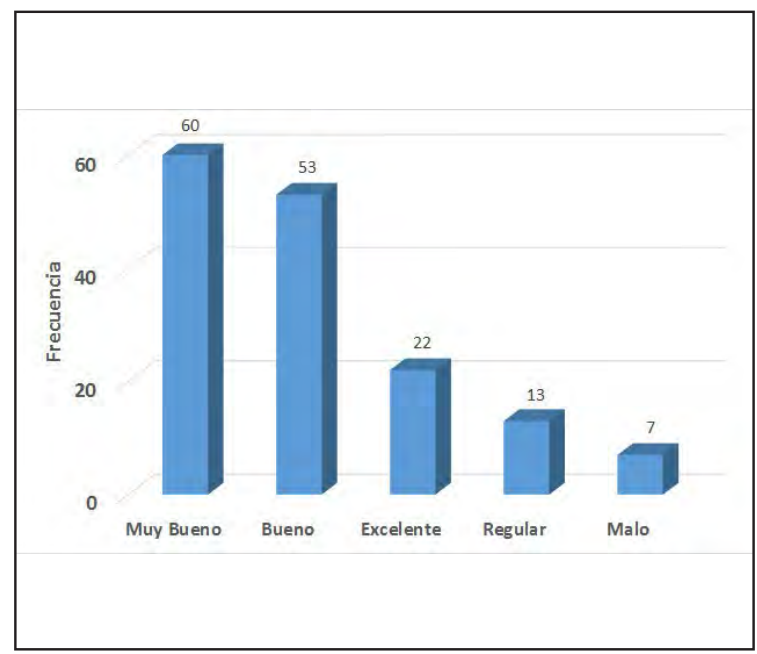

Figura 10. Capacidad para actuar con justicia y realismo.

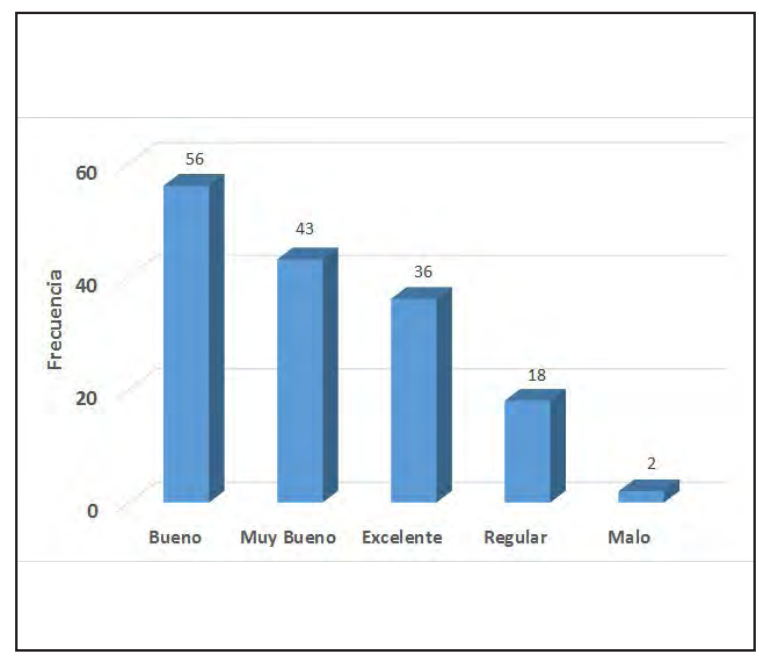

Figura 11 . Nivel de satisfacción con la labor que realiza. de Ingeniería de Minas cumple con este requisito, solo un mínimo porcentaje afirma lo contrario.

La figura 3, reportan resultados positivos y alentadores en el desempeño docente, referente a la comunicación de los profesores con sus estudiantes, pues tanto en el rubro de bueno como muy bueno llegan al $60 \%$, lo que indica que la comunicación entre docentes y estudiantes es bueno.

La figura 4 nos muestran también que en este rubro el desempeño docente en lo referente a la capacidad de planificar el proceso de enseñanza aprendizaje esta considerado como bueno.

El rubro utilización de recursos y materiales también esta considerado como bueno, el desempeño docente, en lo referente a la utilización de recursos y materiales, solo un mínimo porcentaje considera que este rubro el docente no utiliza los medios y materiales en forma adecuada (figura 5).

La figura 6 nos muestra que el desempeño docente ítem, información sobre la marcha del aprendizaje de los estudiantes también esta considerado como buena pues los rubros de bueno y muy buena llegan casi al $80 \%$ de aceptación por parte de los estudiantes.

El rubro mostrado en la figura 7, al igual que los anteriores también esta considerado como bueno, en conclusión la sub variable facilidad en el aprendizaje todos sus rubros están considerados como buenas.

\section{Emocionalidad:}

Mas del $60 \%$ de los encuestados considera que los profesores de la facultad de Ingeniería de Minas tienen vocación (figura 8).

Cerca del $90 \%$ de los encuestados considera que el desempeño docente en el rubro de la autoestima esta considerado como muy bueno, solo un pequeño porcentaje consideran que es malo (figura 9).

El desempeño docente en el rubro Capacidad para actuar con justicia y realismo, también esta considerado como bueno (figura 10).

El rubro mostrado en la figura 11, es considerado como bueno también.

\section{Responsabilidad laboral:}

Más del $90 \%$ de estudiantes consideran que sus profesores asisten puntualmente, lo que se traduce en un buen desempeño laboral (figura 12).

Existe consenso en los estudiantes de la facultad de Ingeniería de Minas, que sus profesores participan en 


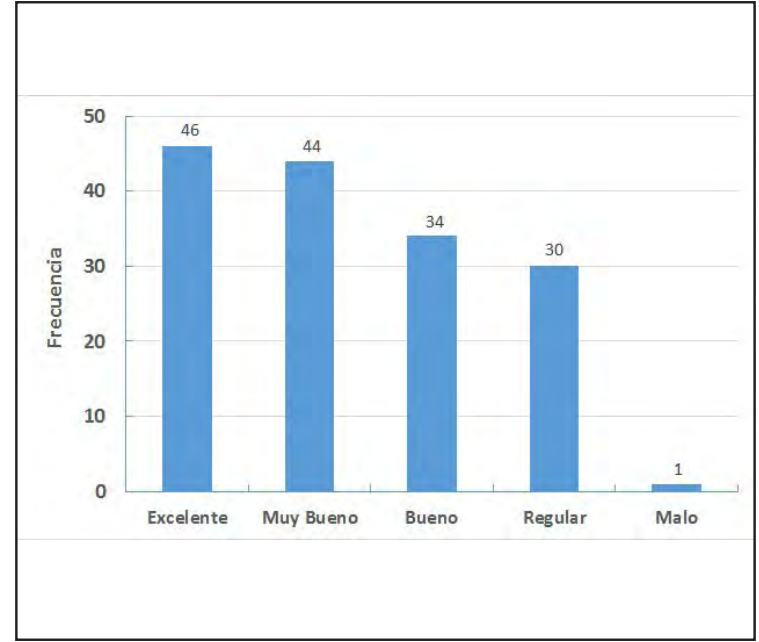

Figura 12. Asistencia y puntualidad.

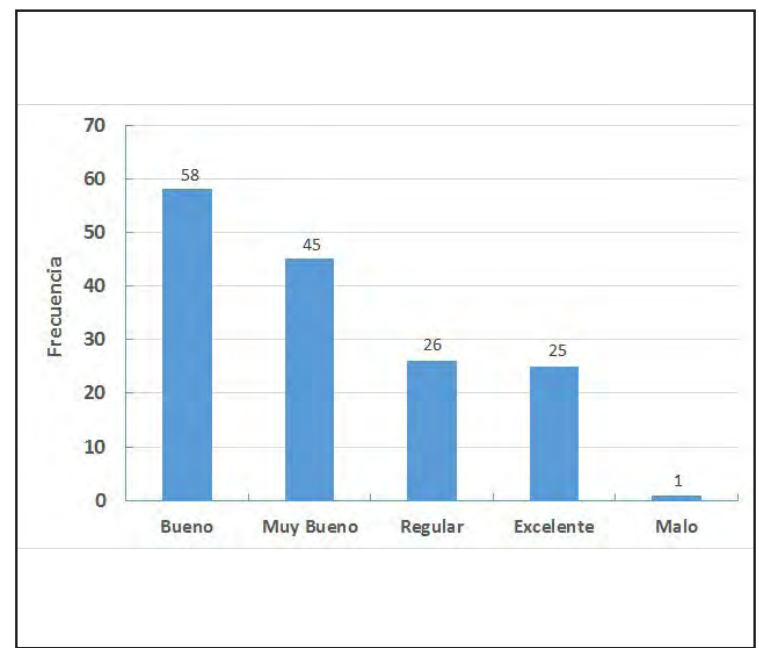

Figura 13. Participación en las sesiones metodológicas y/o jornadas de reflexión entre docentes

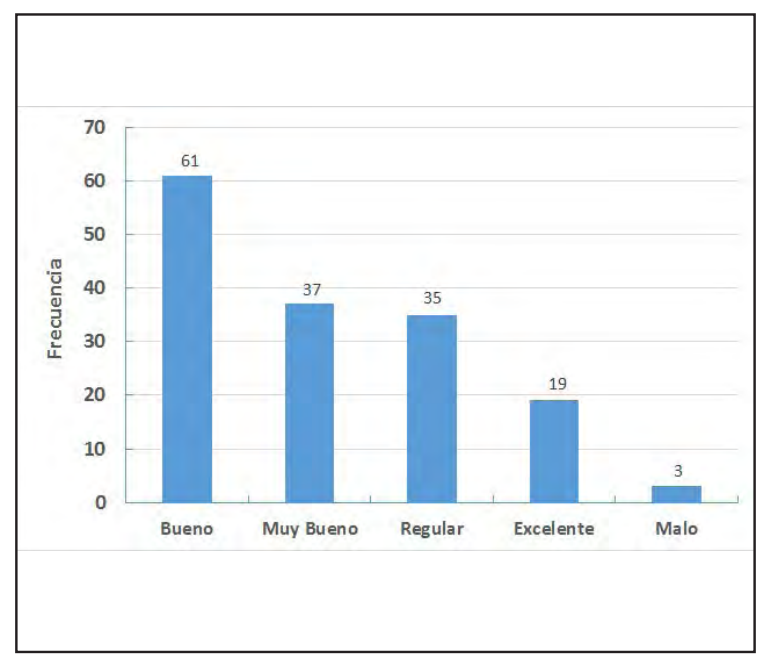

Figura 14. Cumplimiento de la normativa.

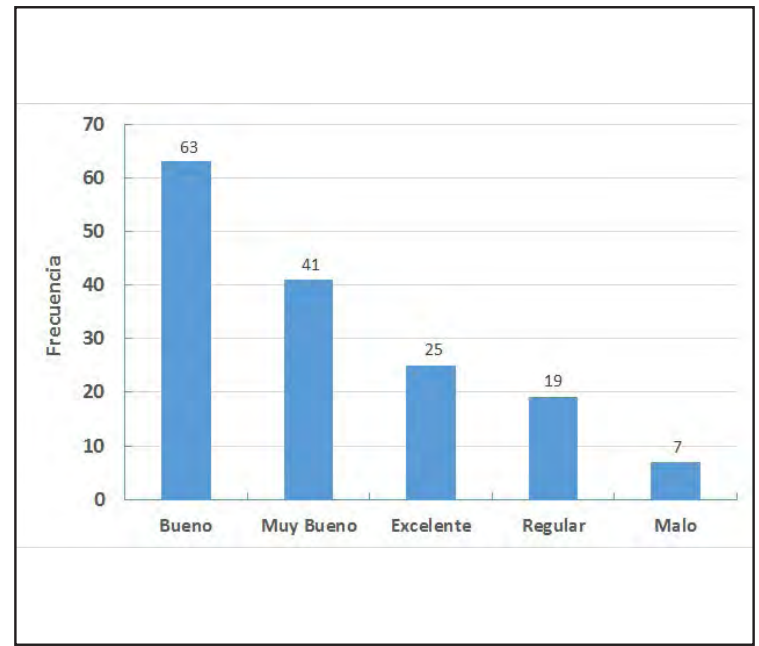

Figura 15. Nivel de preocupación y comprensión de los problemas de sus estudiantes.

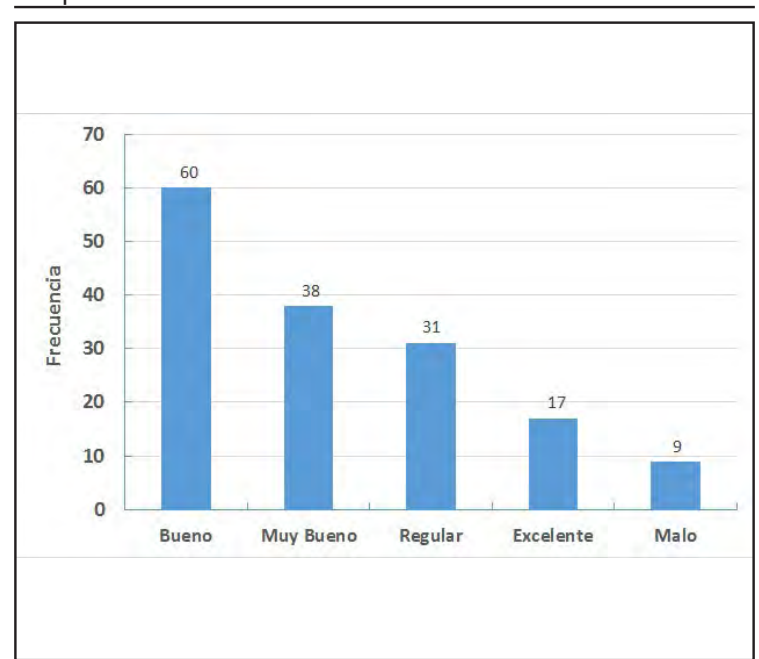

Figura 16. Nivel de expectativas al desarrollo de sus estudiantes.

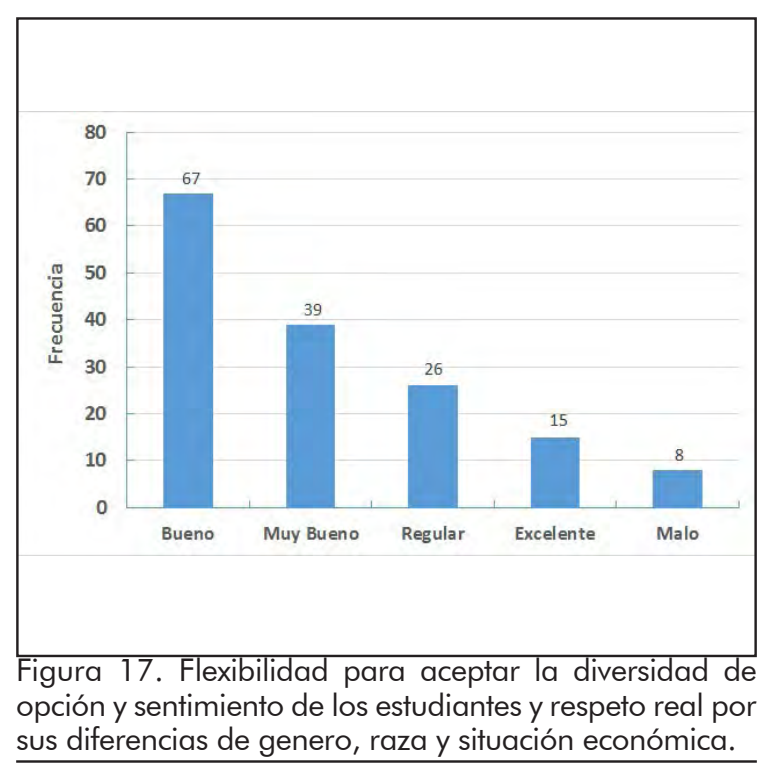


jornadas de reflexión entre los docentes, lo que indica que el desempeño docente en cuanto a responsabilidad laboral es bueno (figura 13).

Más del $80 \%$ de los estudiantes de la facultad de Ingeniería de Minas consideran que sus profesores cumplen con las normativas de su Universidad (figura 14).

\section{Relaciones interpersonales con sus estudiantes:}

El ítem de la figura 15, esta considerado como bueno, es decir que los profesores de la facultad de Ingeniería de Minas de Huancayo, se preocupan de los problemas que tienen los estudiantes.

La figura 16 nos muestra que los profesores de la facultad de Ingeniería de Minas de la Universidad Nacional de Centro tienen cifradas expectativas en sus estudiantes, pues los rubros de bueno y muy bueno pasan del $60 \%$ de los encuestados.

De acuerdo a los resultados mostrados en la figura 17 se nota que también el profesor es flexible con sus estudiantes.

\section{DISCUSIÓN}

El análisis del desempeño docente que corresponde a facilidad del aprendizaje con 7 ítem, emocionalidad con 4 ítems; responsabilidad laboral con 3 ítems; relaciones interpersonales con sus estudiantes con 3 ĺtems y resultados de su labor educativa con 1 solo ítem; en términos generales, podemos considerar que los 18 ítems en evaluación, el $100 \%$ se encuentra con un calificación entre buena, muy buena y excelente, por lo que podemos inferir que en cuanto a desempeño docente, esta variables esta calificada como buena.

Este resultado coincide con lo reportado por Cerdeña (2007), quien evaluando también el desempeño docente y la calidad educativa de La facultad de Ingeniería Química de la Universidad Nacional de la Amazonía Peruana, encontró también una calificación de buena.

En conclusión, el desempeño docente de la facultad de Ingeniería de Minas se encuentra en la calificación de buena, en todos los rubros evaluados.

\section{REFERENCIAS BIBLIOGRÁFICAS}

Asamblea Nacional de Rectores. (2004). Lineamientos de autoevaluación con fines de acreditación escuelas y programas de Post Grado. Lima.

Asamblea Nacional de Rectores. (2004). Estándares para la evaluación con fines de acreditación para las facultades de derecho. Lima.

Bazán León, V. A. (s.f.). Calidad Aplicada a la Educación.

Bretel, L. (2002). Consideraciones y Propuestas para el Diseño de un Sistema de Evaluación del Desempeño .

Cerdeña, P. (2007). Desempeño docente y su relación con la calidad educativa de la Facultad de Ingeniería Química de la Universidad Nacional de la Amazonia Peruana. tesis Maestria, UNAP, lquitos.

Farro Custodio, F. (2004). Evaluación y Acreditación para Universidades de Calidad. Primera edición. Lima: UDEGRAF S.A.

Fernandez Tilve, D. (1999). La Evaluación de la Actividad Docente del Profesorado Universitario: el Caso de la Universidad de Santiago de Compostela. España: Revista electrónica interuniversitaria de formación del profesorado.

Kells, H. (1997). Sistemas Nacionales de Garantía y control de la Calidad académica.

López, J., \& Otros. (1996). Inspección y Centros Educativos. Madrid.

Luján Castro, J., \& Puente Azcutia, J. (s.f.). Evaluación de Centros . Madrid: Ministerio de Educación y Ciencia.

Martín Marin, B. (1999). La evaluación hoy en la Universidad de Extremadura. España: Revista Electrónica Inter-universitaria de Formación del Profesorado.

Messina, G. (1999). Estudio sobre el estado del arte de la investigación acerca de la formación docente en los noventa. Revista Iberoamericana de Educación(19).

Ministerio de Educación y Ciencia. (1994). Centros educativos y calidad de la enseñanza. Madrid: Secretaría de Estado de Educación.

Munévar Molina, R. A. (2000). Organización de Estados Iberoamericanos El desempeño profesional como evaluación cognitiva.

Orden Hoz, A. (1985). Evaluación educativa. Buenos Aires.

Programa de Naciones Unidas. PENUD. (1995). Informe sobre desarrollo humano. PENUD. México.

Sanz, M. (2000). Experiencia educativa: factores de calidad y NNTT (Vol. 164). Comunicación y Pedagogía.

Schmelkes, S. (2002). Departamento de investigaciones educativas del CINVESTAV del Instituto Policlínico Nacional. México. 
Sevillano García , L. (2004). Estrategias innovadoras para una enseñanza de calidad. Madrid: Pearson Educación.

Valdez, D. (2000). "Formación de formadores", documento presentado en la reunión El desempeño de maestros en América Latina: nuevas prioridades. Brasilia.
Vidal Araya, L. (2005). Evaluación Organizacional de la Excelencia Docente. VIII Región, Chile: Centro Educacional de Alta Tecnología. CEAT.

Yarleque Chocas, L. (2007). Investigación en Educación y Ciencias Sociales. Primera Edición Huancayo. Perú. 